\title{
The Research Progress of Undergraduates Resiliency and its Application in Mental Health Education
}

\author{
$\mathrm{Xu} \mathrm{Bin}^{1,2}$, Liang $\mathrm{Fei}^{1^{*}}$ \\ 1) Art School, Guangxi University, Nanning, Guangxi, China (171206923@qq.com) \\ ${ }^{2)}$ College of Tourism and Geographical Sciences, Yunnan Normal University, Kunming, Yunnan, China (xubin5896@qq.com)
}

\begin{abstract}
The researches of resiliency have became hot points for psychology. These researches also have been extended to the students in colleges by many scholars. Aims: This paper scientifically introduces the overseas and domestic research progress of students resiliency in colleges. Methods: analyses the theories, models, measuring scales, generating conditions of colleges students resiliency through comparing overseas and domestic research results. Result: presents some viewpoints that the improving undergraduates resiliency will become a important researching direction of mental health and its concrete implementation ways.
\end{abstract}

Index Terms-Undergraduates, Resiliency, Mental health, Application

\section{大学生心理弹性研究进展及其在心理健康 教育中的应用}

\author{
许斌 梁斐* \\ 广西大学艺术学院, 南宁, 广西, 中国
}

摘 要 心理弹性已成为当前心理学研究的热点问题, 许多学者已经把该研究延伸到了大学生群体。目的: 该文比较系统地介绍 了国内外关于大学生心理弹性的研究进展; 方法: 通过国内外的比较研究分析了大学生心理弹性的理论、测量工具、产生条件和研究 现状; 结果：提出了提升大学生心理弹性将成为未来心理健康教育的重要方向的观点和具体实施路径。

关键词 大学生, 心理弹性, 心理健康, 应用

\section{1. 引言}

\section{1 心理弹性概念的起源和翻译}

弹性 (resilience), 原本是一个物理学中弹性力学的概 念, 是指物体受到外力挤压时的回弹, 即在压力消除后能 迅速反弹回原始状态。人格弹性研究最早始于美国, 至今 有三十多年的历史, 在中国尚处于起步阶段。美国心理学 家 Block 在 1950 年提出了 ego-resiliency（自我心理弹性） 这个概念, 这可能是心理学界第一次使用 Resiliency（心理 弹性) 这个术语。而美国心理学家 Rutter 和 Garmezy 等人 在 20 世纪 70 年代对 Resiliency（心理弹性）的关注为以 后心理弹性的广泛研究奠定了基础 ${ }^{[11}$ 。到了 20 世纪 80 年 代以后, 越来越多的研究发现: 同样是面临高危或压力环 境, 有些个体却能适应良好并能健康发展, 甚至超过了正
常个体的发展或适应水平, 表现出心理弹性[2]。这后来成 为了积极心理学关注的热点 [3]。

\section{2 心理弹性概念的界定}

目前为止, 国内外学者对心理弹性的概念尚无统一定 论, 已有许多学者从不同角度提出了心理弹性的定义。

国外目前的动态是开始把心理弹性看做是一个动态的 过程, 而不是一种可能具有或者不具有的不变的特质 [4]。 心理弹性被认为是人健康发展的一个组成部分, 并能在生 活中不断得到发展和丰富。Masten. Best \& Garmezy 通过对 三种不同的心理弹性群体的描述, 对心理弹性概念进行了 定义: 第一类, 心理弹性是指经历了高危险的人群（如消 防队员、士兵、警察等) 通过克服逆境获得良好的发展, 认 为心理弹性是个体内部的一种个人力量; 第二类, 心理弹 
性是个体面对持续的生活压力 (如父母离异、家庭经济困 难、家庭暴力等挫折) 而能很好地适应这些压力; 第三类, 心理弹性是指经历了创伤后的个体得到了很好的恢复 [5]。

国内学者朱森楠对心理弹性的界定得到了国内同行普 遍的认可, 他认为“心理弹性是个体面对内外压力困境时, 激发内在潜在认知、能力或心理特质,运用内外资源积极修 补、调适机制的过程, 以获取朝向正向目标的能力、历程 或结果”[6]。

\section{2. 心理弹性产生的条件}

尽管各研究者从不同的研究角度对心理弹性进行了定 义, 但所有的研究都公认心理弹性产生需满足两个必不可 少的要素或条件, 即: (1) 个体必须暴露在高危环境或严 重困境下;（2）尽管受到这些威胁或逆境对个体发展的影 响, 但个体仍能保持适应良好。正是这两个要素构成了心 理弹性的本质。因此, 心理弹性是个体人格因素和环境因 素共同作用的结果。一个具有良好人格弹性的人不仅能忍 受逆境或者应付逆境, 而且还能够“弹回（Bounce back）” 所遭受的逆境, 并且在持续的危险和逆境面前仍然能维持 良好的功能水平。

\section{3. 测量心理弹性的工具}

为了进行心理弹性的定量研究, 国内外的学者都根据 不同的维度和文化背景特征编制了心理弹性测试量表。

\section{1 国外量表}

国外的学者针对大学生和成人根据各自所依据的理 论, 编制出了相应的心理弹性测量工具。归结起来, 常见 的有以下两类[7]:

(1)测量个体心理弹性内在保护性因素的工具

(1)特质性自我心理弹性量表: 由 Bartone, Ursano 和 Wright 等人编制, 主要测量个体的耐受力(hardiness)这种人 格倾向, 共 45 个题目, 包括执行、控制和挑战三个子维度。

(2)自我心理弹性量表: Block 和 Kremen 编制的量表, 是一个测量个体的自我心理弹品质的自评量表, 该量表有 14 个题目, 在国际上权威性比较高, 得到了广泛的使用。

(2) 测量个体心理弹性外在和内在保护性因素的工具

(1)成人心理弹性量表(简称 RSA): 挪威学者 Friborg 等 人在总结前人研究的基础上, 编制了成人心理弹性量表。 原始量表由 39 题构成, 分成 6 个维度: 自我认知、未来计 划、家庭凝聚、社会资源、社会能力、计划特点。主要测 量个体在压力情景下维持心理健康和适应良好所具备的保 护机制和因子。RSA 目前在国内外运用最广泛的心理弹性
测量量变之一, 特别是在大学生心理弹性的研究中, 我国 的许多研究者也在使用这个量变。

(2)青少年心理弹性量表(READ): Odin Hjemdal 等人编 制了青少年复原力量表。该量表选取挪威学 Friborg 编制的 成人复原力量表的部分题目修改而成, 有 5 个维度: 个人 能力、社会能力、计划特点、家庭凝聚和社会资源, 共 39 个项目。

\section{2 国内量表}

国内学者编制的心理弹性量表也有自己的特点, 对大 学生研究的针对性更强。主要有:

(1)大学生复原力量表: 阳毅 2005 年编制的大学生复原 力量表, 包括 31 个项目, 共 6 个维度: 自我效能、自我接 纳、稳定性、问题解决、朋友支持和家人支持 [8]。

(2)大学生心理弹性问卷: 徐家华 2010 年编制的该问卷 具有较好的信效度, 它包括 42 个项目, 含 8 个维度: 自我 效能、自我接纳和自我价值、调用心理资源和社会资源的 能力、情绪稳定性、社会交往能力和问题解决能力、朋友 支持和社会支持、家人支持、目标感和执行力[9]。

需要强调的是即使是国内的学者在使用国外量表的时 候大多对这些量表进行了校验和修订, 这样更能适用于国 内的研究对象。

\section{4. 大学生心理弹性的研究现状}

通过使用人心理弹性量表 (简称 RSA), 研究结果显示 中国大学生的心理弹性水平总均分为 2.76(S.D. $=0.41)$, 挪 威的 Hjemdal 采用同一心理弹性问卷测量了挪威大学生的 心理弹性状况, 总均分为 5.31(S.D.=0.66) [10], 说明在某种 程度上国外大学生的心理弹性可能比我国大学生要好。

\section{1 不同性别大学生心理弹性的差异}

男生在自我意识和未来计划两个因子上得分显著高于 女生, 这说明男生在大学里对自我的认识、个人未来的发 展有更多的考虑, 较早地确定了目标。但是, 雷万胜等人 通过调查也发现女大学生的总体心理弹性要高于男大学 生, 在坚韧维度上的差异达到显著水平 ${ }^{\mathrm{I}}{ }^{111}$, 这也反映了我 国妇女沿袭了坚韧的传统美德。在国外的研究中, 加拿大 学者 Hannah 也发现, 在青年时期, 女性比男性要坚韧很多, 但到老年她们的坚韧会减少很多[12]。

\section{2 不同年级大学生心理弹性的差异}

用 RSA 测量不同年级大学生的心理弹性, 发现各年级 学生心理弹性差异不显著。但是, 也有研究发现即将毕业 的学生心理韧性得分最低[11], Hannah 等人的研究也发现, 
坚韧性随着年龄和年级发展, 年龄和坚韧之间呈负相关 [12]。这可能是由于当今社会就业竞争激烈, 大学毕业生 就业压力过大, 与考入大学时的期望值相差甚远, 找工作 时不可避免会遇到许多意想不到的挫折或困难, 经努力尝 试抵抗挫折或困难失败后, 丧失了面对逆境时的有效应对 和适应能力, 消极、悲观情绪导致心理弹性降低。

\section{3 不同经济条件大学生心理弹性的差异}

王燕秋在她的研究中把研究对象按经济情况进行了分 组, 结果发现, 贫困生和非贫困生在心理弹性总分上没有 差异。可见, 经济状况不影响心理弹性, 不是心理弹性的 预测因素, 即无论经济状况好坏, 个体的心理弹性都能够 在后天得到锻炼和提高, 但在社会支持因子上有显著的差 异[10]。几乎所有的研究者都认同社会因素在贫困大学生心 理问题形成中的作用。贫困大学生由于现实经济压力, 常 常远离群体交往。当面对应急事件时, 很少感受到支持, 更易产生焦虑、抑郁、敏感等不良情绪, 这就是他们社会 支持系统不完善的表现。

\section{5. 大学生心理弹性和其他心理健康指标的关系}

目前国内学者已经开始把心理弹性的测量与其他心理 健康指标的测量联系在一起, 研究常选用的量表是: 心理 弹性量表(RSA)、症状自评量表(SCL-90)、青少年生活事件 量表、艾森克个性问卷 $(\mathrm{EPQ}) 、 16 \mathrm{PF}$ 、社会支持量表。从查 阅的大量研究资料来看, 这些量表也都是适合于我国大学 生的常用心理测验工具。以往都是单一量表研究, SCL-90 应用的最多, 而将人格弹性、生活事件、社会支持、人格 特点等综合起来对大学生的研究还是比较少的。这些研究 还运用了相关分析、逐步多元回归、建立回归方程等, 使 研究更具体化。但从近三年的文献检索来看, 这样的研究 已经有了, 说明大学生心理弹性的综合研究方法开始受到 了学界的关注。

近三年国内的研究显示, 心理弹性与外向性、社会支 持有显著的正相关，与神经质、生活事件、有显著的负相 关[10]。进一步进行回归分析表明, 社会支持、内外向、神 经质及负性生活事件对心理弹性能预测 $29.9 \%$, 说明社会 支持、内外向、神经质及生活事件是影响心理弹性的重要 因素。同时, 本研究还发现心理弹性 RSA 测量高分组在社 会支持和内外向上分数较高, 而 RSA 低分组在神经质、生 活事件、SCL-90 的得分较高, 说明心理弹性较差的个体情 绪更不稳定、更易受生活事件的影响、心理健康水平较低。 可见, RSA 能有效区分个体的心理弹性并预测个体心理健 康水平。Hjemdal[13]对挪威大学生的研究也发现, 心理弹 性与生活事件的交互作用能显著的预测心理健康。在国外,
还有学者把心理弹性视为预测个体应激后神经精神障碍的 重要指标。

\section{1 心理弹性与人格显著相关}

Laura[14]等调查了智力圣地亚哥大学的 132 名大学 生, 结果显示: 心理弹性和神经质有显著的负相关, 与内 外向有显著的正相关。Friborg 等[15]将 RSA 问卷应用于研 究 482 名大学生, 结果发现, 外向性、情绪稳定性与 RSA 的各个因子都有显著的相关, 其中外向性与社会能力的相 关最高, 情绪稳定性与自我意识和未来计划的相关最高, 即与个人能力的相关最高。国内王燕秋的研究也得出类似 的结果, RSA 总分及 6 个因子分 EPQ 的内外向呈显著正相 关, 与神经质呈显著负相关, 其中内外向与社会能力相关 最高, 神经质与个人能力相关最高 ${ }^{[101}$ 。这说明: 人格特点 是心理弹性的重要影响因素, 而且在不同的文化下个体的 心理弹性可能受相同因素的影响。

\section{2 社会支持对心理弹性有显著的预测力}

社会支持对心理弹性有显著的预测力, 这在国内与国 外的研究结果上取得了一致。Simon Denny[16] 等调查了新 西兰 100 多所中学的近万名学生的抑郁情况及其心理弹 性, 发现社会支持、家庭关系是心理弹性的影响因素。 Amanda[17] 进行的追踪研究也发现家庭资源能够促进弱 势青年的心理弹性。大量的研究显示, 社会支持是心理健 康的重要保护资源, 社会支持与心理健康水平正相关, 提 高个体的社会支持可以促进个体的心理健康。他把人通过 各种量变的分析将心理弹性影响的因素归为三类: (1)个人 能力和人格特质(Individual-level), 其中能力包括体质、智 力、社交等, 特质包括自尊、自我效能感、控制感、幽默 感等; (2)家庭支持系统(Family-level); (3)社会支持(Social support-level)。

\section{6. 大学生心理弹性在高校心理健康教育中的应用}

心理弹性的理论和测评工具为提升大学生心理弹性提 供了有效路径。在高校, 除了传统的宣传、讲座、构建支 持系统、危机干预等传统心理健康辅导方式, 可以对大学 生适当进行挫折教育。在设计挫折教育时, 要因人而异, 要根据每个个体的具体情况和心理弹性水平而定, 主要取 决于个体的自尊和自信水平。在一些辅导活动的设计中, 挫折性因素设计过多、强度过大, 会导致个体的自信和自 尊水平受到负面影响。但危险性因素设计过少、强度太弱, 又不足以引发个体心理弹性重组, 起不到提高心理弹性水 平的效果。我们提倡在学校这个保护性因素相对稳定可控 的环境内, 设计适度可控的挫折教育, 让挫折和磨难成为 
学生人生的一笔重要财富, 培养大学生百折不挠、顽强拼 搏、不抛弃不放弃的意志品质, 是锻炼和提高大学生心理 弹性水平的有效方法。

\section{参考文献(References)}

[1] Block J and Kremen, IQ and Ego-Resiliency: Conceptual and empirical connections and Separateness. Journal of Personality and Social Psychology, Vol. 70, No. 2, pp. 349-361, 1996.

[2] Rutter M, Resilience in the face of adversity: Protective factors and resistance to psychiatric disorders. British Journal of Psychiatry, Vol. 147, pp. 598-611, 1985.

[3] ZENG Ling-juan, The Thinking of value concerned resiliency theory in mental health education. Academic Forum, Vol. 5, No. 220, pp. 180-183, 2009.

[4] Craig A Olssona, Lyndal Bonda, Jane M Burnsb, Dianne A Vella-Brodrickc, Susan M Sawyerd, Adolescent resilience: A concept analysis. Journal of Adolescence, Vol. 26, No. 1, pp. 1-11, 2003.

[5] Sue How ard, John Dryden and Bruce Johnson, Childhood Resilience: review and critique of literature. Oxford Review of Education, Vol. 25, No. 3, pp. 307-323, 1999.

[6] ZHU Sen-nan, The concept of Teenagers resilience. http://www.bsjh.hcc.edu.tw/guid/guid3.htm, 2003.

[7] ZHANG Jia-jia, Research on the resilience characteristics and resilience improvement of military students. Master thesis, Third Military Medical University, China, 2011.

[8] YANG Yi. The development of the resiliency scale of university students and its application. Master thesis, Central China Normal University, China, 2005.

[9] XU Jia-hua, the development and application in the resilience scale of university students. Master thesis, Fujian Normal
University, China, 2010.

[10] WANG Yan-qiu, the study of psychological resilience and influencial factors of the students from high professional medical schools. Master thesis, Third Military Medical University, China, 2010.

[11] LEI Wan-sheng, CHEN Yi and CHEN Jing-tian, research on resilience of college students. China Journal of Health Psychology, Vol. 16, No. 2, pp. 155-157, 2008.

[12] Hannah TE, Correlates of psychological hardiness in Canadian adolescents .The Journal of Social Psychology, Vol. 127, No. 4, pp. 339-344, 1987.

[13] Hjemdal $\mathrm{O}$ and Friborg O, Resilience Predicting Psychiatric Symptoms:A Prospective Study of Protective Factors and their Role in Adjustment to Stressful Life Events. Clinical Psychology and Psychotherapy, Vol. 13, No. 3, pp. 194-201, 2006.

[14] Campbell-Sills L, Cohan SL and Stein MB, Relationship of resilience to personality, coping, and psychiatric symptoms in young adults .Behaviour Research and Therapy, Vol. 44, No. 4, pp. 585-599, 2006.

[15] Friborg O, Barlaug D, Martinussen M,et al, Resilience in relation to personality and intelligence. International Journal of Methods in Psychiatric Research, Vol. 14, No. 1, pp. 29-42, 2005.

[16] Simon Denny, Terry Fleming and Peter Watson, Risk and resilience factors for depression among high school students in New Zea land, Journal of Adolescent Health, Vol. 34, No. 4, pp. 115-118, 2004.

[17] Amanda Sackera and Ingrid Schoon, Educational resilience in later life: Resources and assets in adolescence and return to education after leaving school at age 16. Social Science Research, Vol. 36, No. 3, pp. 873-896, 2007. 